\title{
Disturbances of ocular movements and blinking in schizophrenia
}

\author{
J A N I C E R. S T E V E N S \\ From the Departments of Neurology and Psychiatry, University of Oregon Health Sciences Center, \\ Portland, Oregon, USA
}

SUMMARY Neurological examination and electroencephalograms and electro-oculograms, recorded by telemetry, from unmedicated patients with acute and chronic schizophrenia demonstrate a number of abnormalities of extraocular movement including staring, abnormal blink rate, absent glabellar reflex, and increase in horizontal eye movements. As potential clues to the pathophysiology of schizophrenia, these disturbances are analysed in relation to anatomical substrate and dopamine modulation of ocular movement, rapid eye movement sleep, and the neurological disorders in which similar disturbances of ocular movement occur.

In distinguishing dementia praecox from other forms of mental illness, Kraepelin (1913) called attention to a number of ocular signs of the disorder, including pupillary abnormalities, staring, nystagmus, abnormal blinking, and blepharospasm. Widespread use of neuroleptic medications has now induced a variety of neurological side effects and sequelae which obscure the incidence of these signs and their relevance to the pathology of the schizophrenias. Because these ocular signs may provide clues to the pathology of the schizophrenia syndrome, it seemed worth while to document them carefully in medication-free patients.

\section{Subjects and methods}

Complete neurological examinations have been performed on 45 patients who were free from medication for periods of one month to four years, and 10 patients who had never received drugs or other physical treatment. Nine patients were seen within a year of diagnosis, 14 had been diagnosed between one and five years, and the remaining 29 patients, longer than five years. The youngest patient was 17 years of age, the oldest, 49 years. Thirty-one were men, 24 were women.

The diagnosis was made by senior staff consensus and required the following five of the seven criteria stipulated by Taylor and Abrams (1975): illness duration greater than six months; clear

Supported by National Institutes of Health Grant 18055.

Address for reprint requests: Department of Psychiatry, University of Oregon Health Sciences Center, Portland, Oregon 97201, USA.

Accepted 23 June 1978 consciousness; delusions, hallucinations, or formal thought disorder; restricted affect; absence of signs ${ }^{\infty} \overrightarrow{.}$ and symptoms sufficient to make a diagnosis of $\vec{D} \vec{\circ}$ affective illness or coarse brain disease. In ad- $\frac{+}{\mathbb{D}} \stackrel{N}{\perp}$ dition to the neurological examination, 36 of the $\frac{\rho}{\circ}$ 의 55 medication-free patients and 12 normal control ${ }^{\circ}-$ subjects have had 2-24 hour electroencephalo- $Z$ grams (EEG) and electro-oculograms (EOG) recorded by radiotelemetry during which spontaneous behaviour was recorded continuously by a trained observer. Bipolar EEGs were recorded from tin or gold disc electrodes attached to the scalp with collodion at central-parietal (C3-P3; C4-P4) and temporal (T3-T5; T4-T6) positions bilaterally. Lateral eye movement was recorded between electrodes placed at the outer canthus of each eye. Vertical movements and blinks were recorded between electrodes placed immediately above and below one eye. Subjects wore a small multiplexed 8-channel transmitter (weight $90 \mathrm{~g}$, Benton Instrument Company) held firmly to the head by a special cap.

During the recording period, patients were in their rooms or the dayroom within a radius of 40 50 yards of the receiver antenna. They slept in their own beds, and carried on their usual hospital routine. Electroencephalographic and EOG signals were recorded on a 7-channel FM tape recorder monitored by an ink-writing polygraph. Receiving and recording devices were centrally placed in a hallway near the ward dayroom. The polygraph was run at a speed of $15 \mathrm{~mm}$ per second and could be correlated continuously with ongoing behaviour and accelerated to $30 \mathrm{~mm}$ per second for 
more detailed examination during periods of unusual behaviour. Frequency modulated tape was recorded at $1 \frac{7}{8}$ inches per second, and portions of the taped record were played back during behavioural events of particular interest and for subsequent analysis. Behavioural observations were recorded by a trained observer on a written $\log$ and were coded by number at the moment of occurrence on a time code placed on one channel of the tape recorder and polygraph, assuring exact correlation between EEG-EOG recording and behavioural events.

\section{Results}

Neurological examination of this group of patients demonstrated that, apart from the characteristic abnormalities of mental state and skeletal motor activity (stereotyped behaviours, rocking, bizarre postures), the most common signs were disturbances of ocular contact and movement. These included staring, abnormal blinking, saccades, and ocular pursuit. Prolonged telemetered EEG-EOG confirmed the clinical evidence of abnormal ocular movements. Although slow or sharp activity appeared over the left or right temporal region of one-third of these patients, these abnormalities could not be associated with the clinical changes nor with the appearance of abnormal ocular activity. In contrast, the oculomotor changes were frequently correlated with abnormalities of perception and attention.

\section{ABNORMALITIES OF EYE CONTACT}

A fixed penetrating stare directed into the examiner's eyes, at a distant point, or avoidance and failure of eye contact were noted in 34 patients. These signs were equally common in acutely and chronically ill individuals, and usually responded promptly to neuroleptic treatment in patients with acute psychosis. Interrogation of the patient rarely provided a clear explanation for staring or diversion of gaze. A few individuals indicated that staring or avoidance of eye contact was a response to perceptual change as indicated by the following examples:

A 21 year old man with chronic paranoid schizophrenia, who stared fixedly at the examiner, explained "your face is turning into an animal."

The mother of a 20 year old schizophrenic girl reported: "when I go to the hairdresser I tell her I'll be right back. When I come back an hour later and walk in she just stares at me like I'm a new person to her. At other times she stares at her father like a stranger and shouts 'Get him out of here, he's doing things to me. Who is he? Get him out!',
A 17 year old boy avoided eye contact because "I'm afraid you'll read my thoughts."

No change in simultaneous EEG accompanied these clinical events.

\section{ALTERED BLINK RATE}

Although both Kraepelin (1913) and Bleuler (1950) remarked upon increased blinking in dementia praecox, quantitative studies of blinking in this illness are lacking. Abnormalities of blinking in this group of unmedicated schizophrenic patients fell into three categories: (1) decreased blinking, staring, and absent eye contact, most commonly seen in acute exacerbations of the disorder; (2) steady increase in blinking at rest in patients with chronic schizophrenia; (3) episodic paroxysms of rapid rhythmical blinking associated with hallucinations, sudden impulsive acts, or deluded speech.

Sixteen patients demonstrated episodes of rhythmic or paroxysmal blinking at rates up to 2.5-3 per second, and seven patients, four of whom had never received neuroleptic treatment, displayed absent or decreased blinking $(0-1$ per minute).

Eight patients showed abrupt change in blink rate during delusional excitement, while speaking in response to hallucinated voices, or during autistic reveries.

A 26 year old former college student, in hospital for nine years with an unrelenting dementia praecox, sat indolently in the dayroom of the hospital blinking steadily two and a half times per second. He reported that he was in a "waking nightmare" of frightening sexual experiences (Fig. 1).

A patient with acute catatonia who was studied before all treatment blinked only two to three times per minute during the day, but exhibited 2-10 second epochs of rhythmic blinking two and a half to three times per second associated with a posture of supplication or deep sighing respiration at intervals during a sleepless night (Fig. 2).

\section{ABNORMAL BLINK (GLABELLAR) REFLEX}

In response to gentle taps of the glabella repeated at one second intervals, most normal adults respond by single blinks to the first two to five taps, then stop blinking unless stimulation is discontinued for 10-20 seconds, after which the response returns and again habituates after repetition (Overend, 1896). Twelve of the 50 patients in this study had no response to glabellar tap, six failed to habituate the reflex after 10 or more taps, and 14 patients displayed paroxysmal bursts of rapid blinking to each tap on repetition. Absent blink reflex was most common in patients with acute exacerbation of psychosis, while persistent or 


\section{A.D. 26 YEAR OLD OO CHRONIC UNDIFFERENTIATED SCHIZOPHRENIA 4/26/76 No Med I Mo}
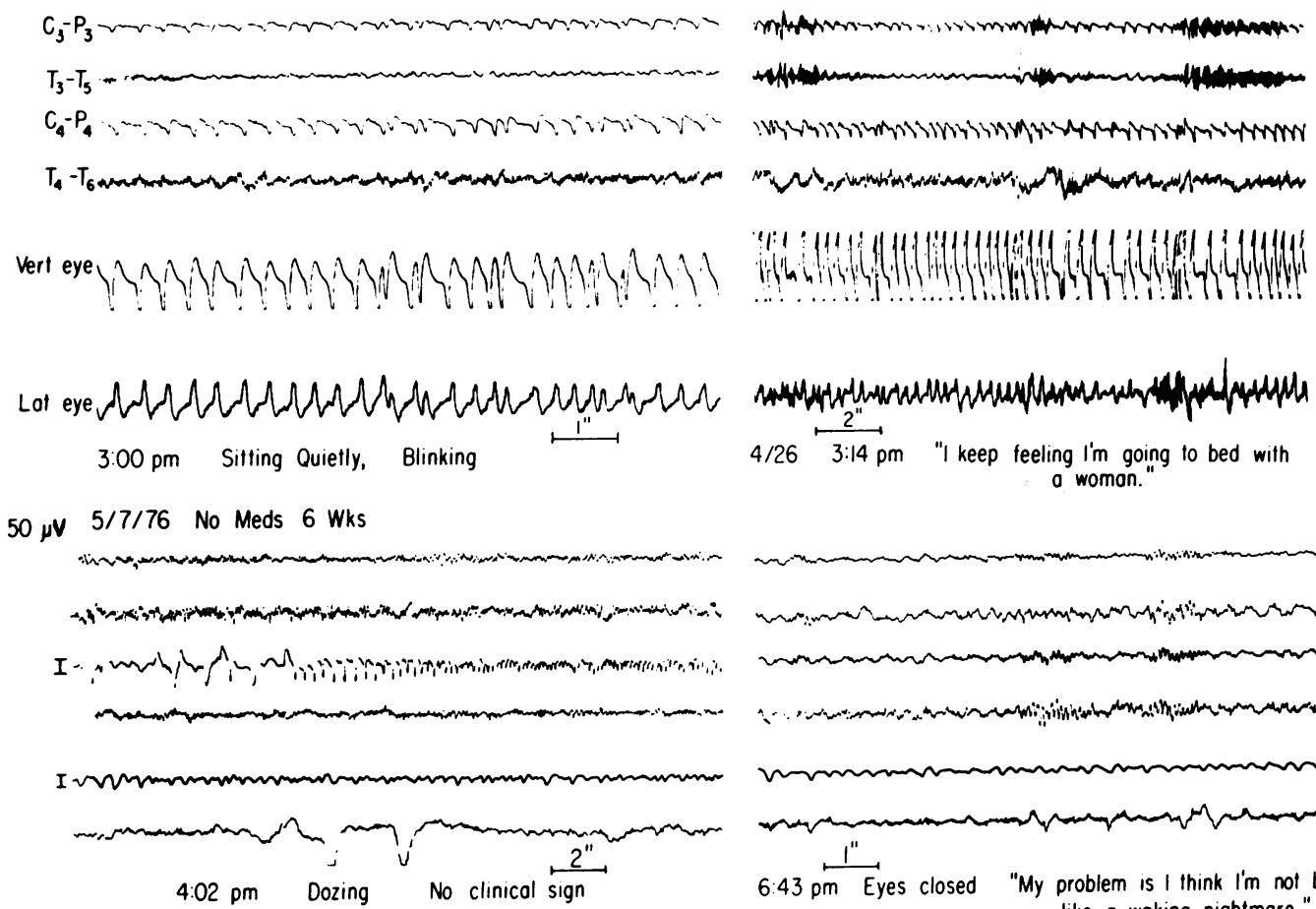

$\mu \mathrm{V}$

$4.02 \mathrm{pm}$ Dozing No clinical sign

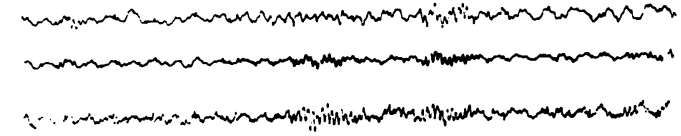

Fig. 1 EEG-EOG by radiotelemetry in a 26 year old man shows steady rhythmic blinking which almost obliterates EEG on the top two panels. Note brief "spike" discharge at 4:02 pm from right parieto-occipital region while patient is dozing. No clinical signs detected. EEG shows asymmetrical temporal function with theta activity on the left, high voltage alpha spindles on the right as patient lies quietly with eyelids fluttering in a "waking nightmare."

paroxysmal response was more characteristic of chronic or less florid psychoses in patients withdrawn from neuroleptics. Six of the 10 patients who had received no previous treatment had no blink reflex to glabellar tap. Return and persistence of the glabellar reflex was one of the earliest effects of neuroleptic drug treatment in these patients.

\section{LATERAL EYE MOVEMENTS}

Abnormalities of three types were recorded: (1) rapid, irregular, searching movements; (2) spontaneous, rhythmic, horizontal saccades; (3) single, sustained, lateral glances.

Darting, rapid, irregular searching movements were exhibited by 12 patients, and were observed most commonly during acute exacerbations of the illness or before all treatment. During such behaviour, patients appeared to be hypervigilant and looking in all directions for the source of auditory or visual stimuli imperceptible to the observer. Characteristically this was accompanied by speech arrest or drivelling. In contrast to psychomotor seizures, which such ocular automatisms may resemble, the darting eye movements of the psychosis usually can be readily, if briefly, interrupted by speaking to or touching the patient. Although the patient can thus be made aware of the activity, an explanation is rarely forthcoming (Fig. 2).

Seven patients, including three without previous treatment, displayed episodes of sustained horizontal lateral oscillations of the eyes at rates of one to two per second lasting for $10-20$ seconds. These rhythmic ocular movements resembled nystagmus in amplitude of excursion and return of eyes to midposition after each lateral movement. Rarely observed during conventional neurological ex- 
Acute catatonia, 21 year old 0 12/30/76

Lights out, searching with eyes

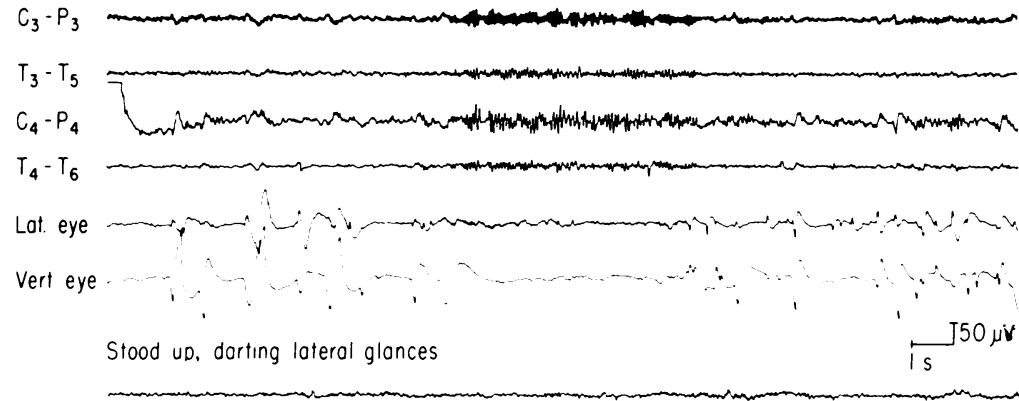

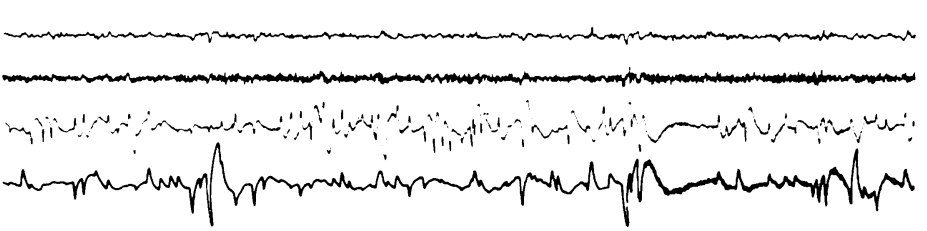

Mute, unable to respond

Fig. 2 Telemetered EEG-EOG from a 21 year old man with acute catatonia for three days with no previous treatment. Top: EEG is unremarkable in dark room at night. Blinking occurs in showers accompanied by lateral eye movements. Middle: 11 am, lighted room. Patient is mute, statuesque, appears frightened. There are rapid, darting, searching, horizontal eye movements. Bottom: midnight in lighted room. Patient is unable to sleep or respond, and is blinking rhythmically and rapidly. EEG shows only low voltage fast

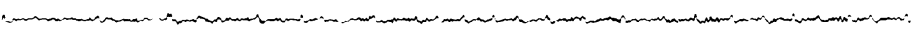
activity.

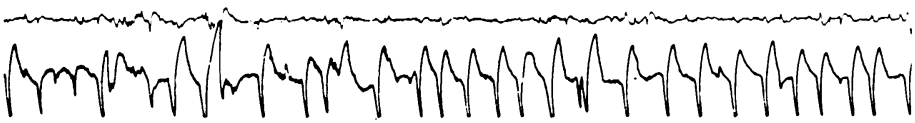

amination, spontaneous horizontal oscillations usually occurred during autistic reveries or psychomotor blocking. These episodes usually lasted for less than 10 seconds, and continued for 30-60 seconds in severely disturbed patients, particularly those with intense delusory and hallucinatory experiences (Fig. 3).

Unexplained single sustained lateral glances interrupted the speech or attention of 14 patients, and, in their most extreme form, constituted a continuous stereotyped automatism in two patients with far advanced schizophrenia.

A 37 year old man in hospital for nearly 20 years after adolescent onset of schizophrenia stood by the hour against the wall in the hospital dayroom repeatedly turning his eyes from side to side as though following a tennis match. Meanwhile he twisted his hands together steadily, flicking his fingers, making signs to himself, smiling and laughing vacuously. On reclining at bedtime his head remained flexed on the neck several inches above the mattress ("psychological pillow"), and lateral eye movements continued for several hours until sleep supervened.

\section{IRREGULAR OCULAR PURSUIT}

Using simple bedside examination of extraocular muscle function in which the patient was asked to fix his eyes on the examiner's moving finger, flashlight, or the movement of his own finger, 24 patients demonstrated one or more abrupt arrests or coarse saccades during each trajectory of horizontal ocular movement. Four patients, all with severe chronic schizophrenia, were entirely unable to follow the moving object with the eyes, and two patients with previously untreated acute schizophrenia repeatedly lost ocular contact with the moving object as their eyes returned from pursuit to staring at the examiner's face. 
F.D. 22 YEAR OLD $\sigma$ CHRONIC UNDIFFERENTIATED SCHIZOPHRENIA 6/2/76 No Med 2 Mos

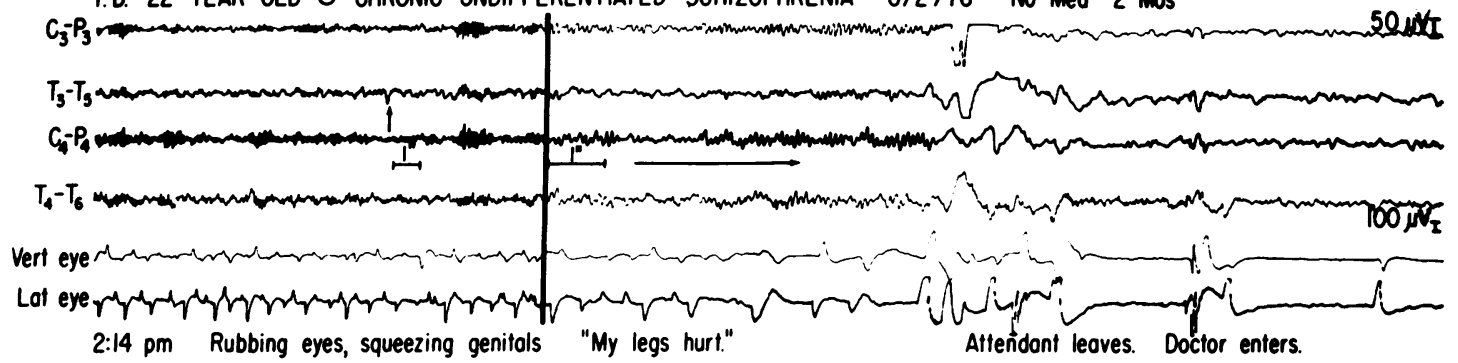

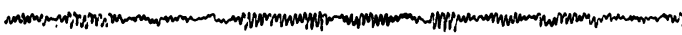

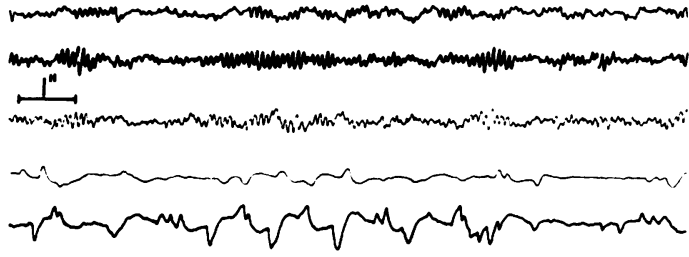

Mosturbotion continues

Stops
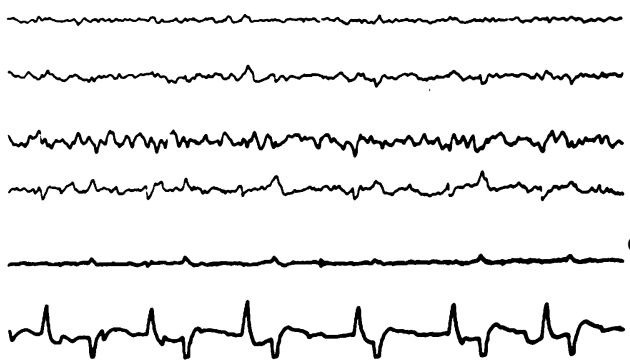

6/3 9:34 pm Quiet, storing

Muttering to self

Fig. 3 EEG-EOG recorded by telemetry from a 22 year old man demonstrates rhythmic 1 cps lateral saccades associated with spindled alpha activity during autistic reverie.

\section{Discussion}

SPONTANEOUS BLINKING: CLINICAL AND EXPERIMENTAL STUDIES

In contrast to normal control subjects, whose blink rates varied from 8-22 per minute (Zametkin et al., 1978), 17 patients in this series had resting blink rates in excess of 60 per minute. Sustained high blink rates were more common in patients with previously treated chronic schizophrenia, while blink rates of less than five per minute were found in five of six patients with catatonic schizophrenia. There are many studies of reflex blinking and the glabellar reflex in the literature, but very few address the clinical significance of spontaneous blinking. Ponder and Kennedy (1928), in one of the few published studies of quantitative blink rate in man, noted that the rate of blinking tends to be remarkably constant in a given individual, and remains essentially unchanged by darkness, temperature, humidity, anaesthesia of the cornea, or deafferentation of the fifth cranial nerve. Observing that one of the earliest features of postencephalitic Parkinsonism was the almost complete absence of blinking, Ponder and Kennedy proposed that normal blinking depends on the integrity of the basal ganglia.
The centres responsible for initiation and modulation of spontaneous blinking are poorlye defined. Van Buren (1963) reported rapid bursts of blinking after stimulation of the caudate nucleus in man. Nashold $(1969,1970)$ elicited eye closure and rhythmic lid flutter in response to stimulation ventral to the superior colliculus and central grey matter, a region from which sensations of burning chest pain and intense emotional reactions were also elicited.

\section{LATERAL SACCADES AND GLANCES}

The darting glances displayed by these patients are strikingly similar to the ocular searching movements termed "checking" displayed by cats and monkeys in strange environments, when intensely frightened, or after administration of amphetamine and cocaine (Ellinwood, 1974). Sato (1977) described visual searching movements after stimulation in the nucleus accumbens in cats. Similar movements are reported in encephalitis, alcoholic encephalopathy, pretectal lesions, and temporal lobe seizures (Mayanagi and Walker, 1974; Escueta et al., 1977). Shimazano et al. (1965) noted that, in contrast to normal subjects who characteristically increase horizontal saccades in response to external stimuli, patients with schizo- 
phrenia decrease or arrest rapid horizontal eye movements in response to the same stimuli. These investigators proposed that the increased horizontal eye movements of schizophrenia, which they termed "waking REM," do not simply reflect arousal but represent a mechanism closely related to the psychotic state.

We observed similar ocular movements after stimulation of the mesolimbic dopaminergic pathway in cats and in response to apomorphine and other dopaminergic agents (Stevens et al., 1974; Stevens and Livermore, 1978). These stereotyped responses were abolished by neuroleptic agents or by elimination of visual stimuli, suggesting that the ocular movements represent dopaminemediated automatisms elicited by visual stimuli (Stevens et al., 1977).

Wallach and Wallach (1964) described episodic rapid regular horizontal oscillations of the eyes at rates of $60-100$ per minute in children and adults with severe forms of schizophrenia. Regularly associated with severe thought disturbance, preoccupation with endogenous percepts, and a sense of personal dissolution, rhythmic lateral saccades in their patients were also abolished by fixation of attention and by remission of the illness. Recurrence of rhythmic saccades coincided with deterioration or recurrence of psychosis.

\section{OCULAR PURSUIT}

First described and illustrated graphically in patients with dementia praecox by Diefendorf and Dodge in 1908, interruption of smooth ocular pursuit movements has more recently been recorded again in a high percentage of patients with schizophrenia and mania (Holzman et al., 1973, 1976; Shagass et al., 1976). Savitsky and Winkelman (1947) described similar cogwheel motion on deviation of the eyes in patients with disease of the corpus striatum, and Luria et al. (1966) reported similar saccades in patients with frontal lobe lesions and severe cognitive disorders. Interruption in smooth pursuit can be overcome partially by increasing the patient's attention to the task (Shagass et al., 1976).

SCHIZOPHRENIA, DOPAMINE BLOCKADE, AND THE LIMBIC SYSTEM

Evidence that the effectiveness of neuroleptic agents against the symptoms of schizophrenia is related directly to the capacity of these agents to block dopamine receptors in the brain has implicated central dopaminergic systems in the pathophysiology of schizophrenia (Carlsson and Lindquist, 1963). Striking similarities between the subjective disturbances and stereotypes of schizophrenia, and the auras and automatisms of psychomotor epilepsy suggest that schizophrenia, like psychomotor epilepsy, is associated with abnormal function in the limbic system (Stevens, 1973). The dopamine innervation of the limbic system derives principally from the most medial units of the pars compacta of the substantia nigra (SN), and from the ventral tegmental area (VTA, A10), located just medial and rostral to SN. From VTA, axons of the dopaminergic neurones of the mesolimbic system ascend to the nucleus accumbens, olfactory tubercle, nucleus of stria terminalis, diagonal band, lateral septum, and the central nucleus of the amygdala (Ungerstedt, 1971). Abnormal electrical activity has been reported from these regions in schizophrenic patients by Heath (1954), Hanley et al. (1970), and others. In keeping with a more medial position in the forebrain, the nucleus accumbens projects to more axial structures of the diencephalon and mesencephalon than the caudate nucleus, including the paramedian pontine reticular formation and tectum (Domesick et al., 1976), regions closely associated with regulation of extraocular movement, arousal and attention.

Species specific stereotyped exploratory behaviours released by dopaminergic agents, consisting of sniffing, licking, and chewing in rodents, depend on the integrity of the neostriatum (Randrup and Munkvad, 1967), while increased exploratory activity induced by these agents is attributed to activation of the nucleus accumbens and other components of the limbic striatum (Iversen, 1977). The disturbances in eye movement demonstrated by schizophrenic patients are usually attributed to the heightened state of fear or arousal associated with the acute psychosis. However, the occurrence of these disturbances in patients with longstanding psychosis, the modulation of these signs by agents which alter central dopamine function, and the appearance of similar signs after stimulation of the mesolimbic dopaminergic system in experimental animals and during temporal lobe seizures of man suggest that these ocular signs may represent abnormal function of the mesolimbic dopamine system.

I express my appreciation to Dr John Lipkin, US Veterans Administration Hospital, Portland, Oregon, to Dr Duane Denney, University of Oregon Health Sciences Center, Portland, Oregon, to Dr Richard Wyatt, National Institutes of Health, Washington, DC, to Dr Ernst Rodin, Lafayette Clinic, Detroit, Michigan, to Dr Gerald Klerman, Eric Lindemann Mental Health Center, Boston, Massachusetts, and to the staffs and 
patients of these hospitals. This study was supported by National Institutes of Health Grant 18055.

\section{References}

Bleuler, E. (1950). Dementia Praecox or the Group of Schizophrenias, p. 22. Translated by J. Zinkin. International Universities Press: New York.

Carlsson, A., and Lindquist, M. (1963). Effect of chlorpromazine or haloperidol on the formation of 3-methoxytyramine and normetanephrine in mouse brain. Acta Pharmacologica et Toxicologica, 20, 140-144.

Diefendorf, A. R., and Dodge, R. (1908). An experimental study of the ocular reactions of the insane from photographic records. Brain, 31, 451.

Domesick, V. B., Smith, G. P., and Nauta, W. J. H. (1976). Contrasting projections of the nucleus accumbens and caudato-putamen in the rat. Procedures of the American Anatomy Society, Lexington, Kentucky.

Ellinwood, E. H. (1974). Behavioral and EEG changes in the amphetamine model of psychosis. In Neuropsychopharmacology of Monoamines and Their Regulatory Enzymes, pp. 281-295. Edited by E. Usdin. Raven Press: New York.

Escueta, A. V., Kunze, U., Waddell, G., Boxley, J., and Nadel, A. (1977). Lapse of consciousness and automatisms in temporal lobe epilepsy: a videotape analysis. Neurology (Minneapolis), 27, 144-155.

Hanley, J., Berkhout, J., Crandall, W. R., Rickles, W. R., and Walter, R. D. (1970). Spectral characteristics of EEG activity accompanying deep spindling in a patient with schizophrenia. Electroencephalography and Clinical Neurophysiology, 28, 90.

Heath, R. G. (1954). Studies in Schizophrenia. Harvard University Press: Cambridge.

Holzman, P. S., Proctor, L. R., and Hughes, D. W. (1973). Eye-tracking performance in psychiatric schizophrenia. Science, 181, 179-181.

Holzman, P. S., Levy, D. L., and Proctor, L. R. (1976). Smooth pursuit eye movements, attention, and schizophrenia. Archives of General Psychiatry, 33, 1415-1420.

Iversen, S. D. (1977). Striatal function and stereotyped behavior. In Psychobiology of the Striatum, pp. 99-118. Edited by A. R. Cools, A. H. M. Lohman, and J. H. L. van den Bercken. Elsevier/NorthHolland Biomedical Press: Amsterdam.

Kraepelin, E. (1913). Lectures on Clinical Psychiatry, p. 24. William Wood and Company: New York.

Luria, A. R., Karpov, B. A., and Yarbuss, A. L. (1966). Disturbances of active visual perception with lesions of the frontal lobes. Cortex, 2, 202-212.

Mayanagi, Y., and Walker, A. E. (1974). Experimental temporal lobe epilepsy. Brain, 97, 423-446.

Nashold, B. S., Wilson, W. P., and Slaughter, D. G. (1969). Sensations evoked by stimulation in the midbrain of man. Journal of Neurosurgery, 30, 14-24.
Nashold, B. S. (1970). Ocular reactions from brain stimulation in conscious man. Neuro-Ophthalmology, 5, 92-103.

Overend, W. (1896). Preliminary note on a new cranial reflex. Lancet, 1, 619.

Ponder, E., and Kennedy, W. P. (1928). On the act of blinking. Quarterly Journal of Experimental Physiology, 18, 89-110.

Randrup, A., and Munkvad, I. (1967). Stereotyped activities produced by amphetamine in several animal species and man. Psychopharmacologia, 11, 300.

Sato, Mitsumoto (1977). Functional changes in the caudate and accumbens nuclei during amygdaloid and hippocampal seizure development in kindled cats. Folia Psychiatrica et Neurologica Japonica, 31, 501-512.

Savitsky, N., and Winkelman, N. W. (1947). Cogwheel phenomenon of the eyes: its clinical significance. Archives of Neurology and Psychiatry (Chicago), 57, 362-368.

Shagass, C., Amadeo, M., and Overton, D. A. (1976). Eye tracking performance and engagement of attention. Archives of General Psychiatry, 33 121-125.

Shimazano, Y., Ando, K., Sakamoto, S., Tanaka, T., Eguchi, T., and Nakamura, H. (1965). Eye movements of waking subjects with closed eyes. A comparison between normals and chronic schizophrenics. Archives of General Psychiatry, 13, 537-543.

Stevens, J. R. (1973). An anatomy of schizophrenia? Archives of General Psychiatry, 29, 177-189.

Stevens, J. R., Wilson, K., and Foote, W. (1974). GABA blockade, dopamine and schizophrenia: ex perimental studies in the cat. Psychopharmacologia, 39, $105-119$.

Stevens, J. R., Livermore, A., and Cronan, J. (1977). Effects of deafening and blindfolding on amphetamine induced stereotypy in the cat. Physiology and Behavior, 18, 809-812.

Stevens, J. R., and Livermore, A. (1978). Kindling of the mesolimbic dopamine system: animal model of psychosis. Neurology (Minneapolis), 28, 36-46.

Taylor, M. A., and Abrams, R. (1975). A critique of the St Louis psychiatric research criterion for schizophrenia. American Journal of Psychiatry, 132, 1276-1280.

Ungerstedt, U. (1971). Stereotaxic mapping of the monoamine pathways in the rat brain. Acta Physiologica Scandinavica, Supplement 367, 1-48.

Van Buren, J. M. (1963). Confusion and disturbance of speech from stimulation in vicinity of the head of the caudate nucleus. Journal of Neurosurgery, 20, 148-157.

Wallach, M. B., and Wallach, S. S. (1964). Involuntary eye movement in certain schizophrenics. Archives of General Psychiatry, 11, 71-73.

Zametkin, A., Stevens, J. R., and Pittman, R. (1978). Ontogeny of spontaneous blinking and of habituation of the blink reflex. Annals of Neurology. In press. 